\title{
DEVELOPMENT OF ZOLMITRIPTAN MOUTH DISSOLVING FILMS: FORMULATION VARIABLES, MECHANICAL PROPERTIES, AND IN VITRO DRUG RELEASE STUDIES
}

\author{
SUDHIR MADDELA ${ }^{1}$, BUCHI N. NALLURI ${ }^{2 *}$ \\ ${ }^{1}$ Department of Pharmacy, Krishna University, Machilipatnam - 521 001, Andhra Pradesh, India. ${ }^{2}$ Siddhartha Pharma Innovation \\ and Incubation Centre, KVSR Siddhartha College of Pharmaceutical Sciences, Vijayawada - 520 010, Andhra Pradesh, India. \\ Email: buchinalluri@yahoo.com
}

Received: 30 January 2019, Revised and Accepted: 15 March 2019

ABSTRACT

Objective: The objective of the present investigation is to prepare zolmitriptan (ZOL) mouth dissolving films (MDFs) and to investigate the influence of formulation variables on physicomechanical, chemical, and drug release properties of the prepared MDFs.

Methods: The MDFs were prepared by solvent casting technique using wet film applicator. The impact of hydroxypropyl methylcellulose of different viscosity grades (hydroxy propyl methyl cellulose [HPMC] E3, E5, and E15), plasticizers (glycerol and polyethylene glycol [PEG]-400), and solubilizing agents (polyvinyl pyrrolidone [PVP K30] and sodium lauryl sulfate [SLS]) on physicomechanical, chemical, and drug release properties were evaluated. The MDFs were also characterized by Fourier-transform infrared spectroscopy, differential scanning calorimetry, and X-ray diffractometry studies.

Results: The MDFs prepared were transparent and smooth and showed no recrystallization. The tensile strength of the MDFs increased significantly with an increase in polymer viscosities, and about a 2.63-fold increase in tensile strength was observed for HPMC E15 MDFs compared to E3, whereas an increase in film thickness resulted in brittle MDFs with low tensile strength. Similar results were observed with percent elongation and folding endurance of the MDFs. In vitro, drug release studies indicate that higher film thickness and polymer viscosities delayed the MDF disintegration and, in turn, the ZOL release. Addition of PVP K30 and SLS to HPMC E3 formulations resulted in 1.66- and 1.53-fold increase in ZOL release rates.

Conclusion: Overall,F7 formulation showed quicker disintegration (within $11 \mathrm{~s}$ ) and ZOL release rates (within $180 \mathrm{~s}$ ) along with good physicomechanical properties. These results indicated that the disintegration and drug release of ZOL can be enhanced to a greater extent by optimizing formulation variables in MDFs.

Keywords: Formulation variables, Mouth dissolving films, Tensile strength, Wet film applicator, Zolmitriptan.

(c) 2019 The Authors. Published by Innovare Academic Sciences Pvt Ltd. This is an open access article under the CC BY license (http://creativecommons. org/licenses/by/4. 0/) DOI: http://dx.doi.org/10.22159/ajpcr.2019.v12i4.32176

\section{INTRODUCTION}

The design of age-specific dosage forms stays to be a challenging task due to the wide range of pharmaceutical and clinical lookouts that must be considered in the design of dosage forms [1]. Palatability and ease of swallowing are imperative factors that are to be considered during the design of dosage forms, especially to pediatrics, who have distinct inclinations and swallowing capabilities [2].

Conventional oral drug delivery systems such as tablets and capsules may not fulfill the necessities of pediatric and geriatric patients due to their differential abilities in swallowing the dosage forms $[3,4]$. In this context, the novel mucosal delivery systems have gained popularity in which the mouth dissolving films (MDFs) are the new and novel drug delivery systems for the peroral delivery of drugs to overcome patient impediments [5], and on contact with saliva, it dissolves within a few seconds without the need of the water making them particularly suitable for pediatric and geriatric patients [6].

Zolmitriptan (ZOL) is a serotonin agonist used for the treatment of migraine [7]. ZOL undergoes hepatic first-pass metabolism, and its absolute bioavailability is $40-50 \%$ of the administered dose with a halflife of 2.5-3 h [8]. At present, ZOL is marketed as oral disintegrating $\left(\right.$ ZOMIG-ZMT $\left.^{\circledR}\right)$ and immediate release tablets $\left(\mathrm{ZOMIG}^{\circledR}\right)$. Formulation of ZOL as MDFs may address the issues of low bioavailability along with providing the quicker onset of actions. Hence, keeping in perspective of the patient compliance and need of the better therapeutic efficacy when compared to the existing marketed formulations, in the present investigation, an attempt was made to deliver the ZOL as MDFs. Moreover, no work was published so far detailing the influence of formulation variables such as film thickness, plasticizers, and polymer viscosities on physicomechanical properties such as tensile strength, the percent elongation of ZOL MDFs along with thorough evaluation on drug loading effect on crystallization, and characterization using photographic, differential scanning calorimetry (DSC) and X-ray diffractometry (X-RD) studies. Hence, the present investigation was aimed at the formulation and evaluation of ZOL MDFs for physicomechanical, chemical, and drug release properties.

\section{MATERIALS AND METHODS}

Materials

ZOL was obtained from Mylan Laboratories, Hyderabad. HMPC E3, E5, and E15 were obtained from Colorcon Asia Ltd., India. Methanol, polyvinyl pyrrolidone (PVP K30), and sodium lauryl sulfate (SLS) were purchased from Loba Chemie, Mumbai. Aspartame and pineapple flavor were obtained from Darwin Laboratories, Vijayawada. All the ingredients of analytical grade were used.

\section{Methods}

Preparation of artificial saliva

Accurately weighed quantities of sodium chloride $-0.844 \mathrm{~g}$, potassium chloride - $1.2 \mathrm{~g}$, calcium chloride dihydrate $-0.193 \mathrm{~g}$, magnesium chloride hexahydrate $-0.111 \mathrm{~g}$, and potassium phosphate dibasic $0.342 \mathrm{~g}$ were added one by one to $500 \mathrm{~mL}$ of distilled water, and then, the volume was made up to $1000 \mathrm{~mL}$ using the distilled water. The $\mathrm{pH}$ was adjusted with $0.1 \mathrm{~N} \mathrm{HCl}$ to 5.7 [9]. 


\section{Preparation of ZOL MDFs}

Initially, placebo MDFs with different polymers and plasticizers were prepared to select a suitable combination that forms films with desired mechanical strength and appearance. After selecting the possible combinations from the placebo studies, ZOL MDFs were prepared as per formula given in Table 1 to a batch size of $5 \mathrm{~g}$. To prepare the films, ZOL was dissolved in a mixture of solvents (water and methanol) in a beaker, followed by the addition of plasticizers and other ingredients under continuous stirring. Finally, the polymer was added and mixed thoroughly. The mixture was sonicated for $2 \mathrm{~min}$ to remove entrapped air bubbles and casted on a glass plate with a wet film applicator set at $30 \mathrm{mil}(750 \mu \mathrm{m})$ and $40 \mathrm{mil}(1000 \mu \mathrm{m})$ thickness. The films were dried at $40^{\circ} \mathrm{C}$ for $60 \mathrm{~min}$ in a hot air oven. The dried films were then peeled off from the glass plate and stored in a desiccator until use.

\section{Chromatographic conditions for the analysis of ZOL}

A HPLC system (Shimadzu) comprising a degasser (DGU-20A3), binary pump (LC- $20 \mathrm{AD}$ ), autosampler (SIL-20 ACHT), and PDA-detector (SPD M20A) was used for the analysis of ZOL. LC solution software was used to collect and process the data, and separation was achieved on Agilent Eclipse Column C18 (150 mm $\times 4.6 \mathrm{~mm}, 5 \mu \mathrm{m})$. Mobile phase consisting of formic acid $(0.02 \% \mathrm{v} / \mathrm{v})$ :methanol $(78: 22 \mathrm{v} / \mathrm{v})$ was used at a flow rate of $1 \mathrm{~mL} / \mathrm{min}$, and the eluents were monitored at $221 \mathrm{~nm}$. The developed method was validated as per ICH guidelines and used for the analysis of ZOL.

\section{DSC studies}

DSC studies for ZOL and selected ZOL MDFs were carried out using DSC (Shimadzu, DSC-60, Japan). Samples weighing $5 \mathrm{mg}$ were sealed in aluminum pans and heated from 50 to $400^{\circ} \mathrm{C}$ at a rate of $10^{\circ} \mathrm{C}$ per min. An empty aluminum pan was used as reference [10].

\section{X-RD studies}

The crystallographic properties of ZOL and ZOL MDFs were studied using X-RD studies. Studies were performed using X-Ray Diffractometer (Shimadzu, XRD-7000, Japan) with $\mathrm{Cu}-\mathrm{K} \alpha$ radiation at $40 \mathrm{kV}$ and $30 \mathrm{~mA}$. $\mathrm{X}$-Ray diffraction patterns were collected over $2 \theta$ range of $10-40^{\circ}$ at a scan rate of $4^{\circ}$ per min. The position and intensities of diffraction peaks were considered for the identification of ZOL in different samples [10].

\section{Fourier-transform infrared (FT-IR) studies}

The compatibility between ZOL and excipients used in MDFs was evaluated using FT-IR studies. Studies were carried out using an attenuated total reflectance (ATR)-FTIR spectrometer (Bruker, Germany). ATR spectra were measured over the wave number range of $4000-500 \mathrm{~cm}^{-1}$ at a resolution of $1.0 \mathrm{~cm}^{-1}$. The powder or film sample was simply placed onto the ATR crystal, and the sample spectrum was collected [10].

\section{Evaluation of ZOL MDFs}

\section{Morphological properties}

Properties such as homogeneity, color, transparency, and surface of ZOL MDFs were tested visually. All the formulations were stored at room temperature $\left(25 \pm 2^{\circ} \mathrm{C}\right)$ with a relative humidity of approximately $65 \pm$ $5 \%$ and were tested periodically every month for a period of 6 months. The films were packed in aluminum foil pouches.

\section{Thickness}

The thickness of the film was evaluated using a screw gauge with a range of $0-10 \mathrm{~mm}$ and revolution $0.001 \mathrm{~mm}$. Anvil of the thickness gauge was turned and the film was inserted after making sure that the pointer was set to zero. The film was held on the anvil and the reading on the dial was noted down [10]. The estimations were carried out in triplicate.

\section{ZOL content}

Three $1 \mathrm{~cm}^{2}$ films were taken from the top, middle, and bottom areas of the film and placed in three individual $10 \mathrm{~mL}$ volumetric flasks containing $5 \mathrm{~mL}$ of distilled water and dissolved. The final volume was made up to the mark with distilled water. The samples were then suitably diluted with artificial saliva and analyzed by HPLC method for ZOL content in MDFs.

\section{Variation of mass}

Mass variation among the different batches of the formulations was calculated by measuring the mass of $1 \mathrm{~cm}^{2}$ film cut from different places of the films [10]. The estimations were carried out in triplicate.

\section{In vitro disintegration studies}

In the present investigation, in vitro disintegration studies were carried out using two independent methods, namely the drop and Petri dish methods [11]. For both the methods, only a small amount of medium was needed, so natural conditions could be simulated.

\section{Drop method}

MDFs of $1 \mathrm{~cm}^{2}$ were placed on a glass slide and placed planar on a Petri dish, and one drop of distilled water was added using a pipette onto the MDF. The time taken for the drop to dissolve the film and form a hole in it was measured. The estimations were carried out in triplicate.

\section{Petri dish method}

In this method, $2 \mathrm{~mL}$ of distilled water was placed in a Petri dish and a film of $2 \times 2 \mathrm{~cm}^{2}$ was placed on the surface of the water, and the time required to dissolve the film completely was measured. The estimations were carried out in triplicate.

Table 1: Composition of different ZOL MDFs

\begin{tabular}{|c|c|c|c|c|c|c|c|c|c|c|c|c|}
\hline \multicolumn{13}{|l|}{ Formulae (5 g size) } \\
\hline Ingredients (mg) & F1* & F2* & F3 & F4 & F5 & F6 & F7 & F8 & F9 & F10 & F11 & F12 \\
\hline \multirow[t]{2}{*}{ ZOL } & 100 & 100 & 100 & 100 & - & - & - & - & - & - & - & - \\
\hline & - & - & - & - & 62.5 & 62.5 & 62.5 & 62.5 & 62.5 & 62.5 & 62.5 & 62.5 \\
\hline HPMC E3 & 375 & 375 & 375 & 375 & 375 & 375 & 375 & 375 & - & - & - & - \\
\hline HPMC E5 & - & - & - & - & - & - & - & - & 375 & 375 & 375 & - \\
\hline HPMC E15 & - & & - & - & - & - & - & - & - & - & - & 375 \\
\hline PEG $400^{\wedge}$ & 25 & - & 25 & - & - & 25 & 25 & 25 & 25 & 25 & 25 & 25 \\
\hline 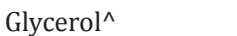 & - & 25 & - & 25 & 25 & - & - & - & - & - & - & - \\
\hline PVP K30 & - & - & 2 & 2 & - & - & 2 & - & - & 2 & & - \\
\hline SLS & - & - & - & - & - & - & - & 2 & - & - & 2 & - \\
\hline Water ${ }^{\wedge}$ & 1730 & 1730 & 1728 & 1728 & 1767 & 1767 & 1765 & 1765 & 1767 & 1765 & 1765 & 1765 \\
\hline Pineapple flavor & 10 & 10 & 10 & 10 & 10 & 10 & 10 & 10 & 10 & 10 & 10 & 10 \\
\hline Aspartame & 10 & 10 & 10 & 10 & 10 & 10 & 10 & 10 & 10 & 10 & 10 & 10 \\
\hline Methanol^$^{\wedge}$ & 2750 & 2750 & 2750 & 2750 & 2750 & 2750 & 2750 & 2750 & 2750 & 2750 & 2750 & 2750 \\
\hline
\end{tabular}

${ }^{*} \mathrm{~F} 1$ and F2 were also casted with 40 mil thickness and represented as F1 (A) and F2 (A), ^Amount taken based on their density. ZOL: Zolmitriptan,

MDF: Mouth-dissolving films, HPMC: Hydroxypropyl methylcellulose, PEG: Polyethylene glycol, PVP K30: Polyvinyl pyrrolidone, SLS: Sodium lauryl sulfate 
Tensile strength

Tensile strength is the maximum stress applied to a point at which the film specimen breaks[12]. In the present investigation, a tensile strength of MDFs was measured using Mini Tech Tensiometer-UTM9051 (Dak Systems Inc., Mumbai, India) fitted with a load cell of $500 \mathrm{~N}(50 \mathrm{~kg}$ ) capacity and the data were collected using Test Bench II software. MDFs of appropriate thickness with fixed dimensions (LxW-10 $\mathrm{cm} \times$ $2 \mathrm{~cm}$ ) were fixed between pneumatic grips with a gauge dimension of $3 \mathrm{~cm}$ length between grips. All the dimensions were entered into the software to calculate the cross-sectional area. The MDF was carefully placed in between the pneumatic grips without any loose folds. The instrument was operated at a speed of $5 \mathrm{~mm} / \mathrm{min}$ until the film breaks. The whole experiment was carried out in triplicate.

\section{Percentage elongation}

In the present study, percentage elongation was computed form TestBench-II software during the tensile strength experiments. The estimations were carried out in triplicate.

\section{Folding endurance}

Folding endurance is determined by repeated folding of the film at the same place until the film breaks. This gives an indication of the brittleness of the film. The number of times the film is folded without breaking is computed as the folding endurance value [13]. The estimations were carried out in triplicate.

\section{In vitro drug release studies}

The in vitro drug release studies were conducted using $500 \mathrm{~mL}$ of artificial saliva as dissolution medium using USP Type $\mathrm{V}$ dissolution rate testing apparatus [14]. A temperature of $37^{\circ} \mathrm{C}$ and $50 \mathrm{rpm}$ were maintained. Each film of appropriate size $\left(3 \times 2.4 \mathrm{~cm}^{2}\right)$ equivalent to $5 \mathrm{mg}$ dose was cut and placed on a watch glass covered with Nylon Wire Mesh. The watch glass was then dropped into dissolution flask. Samples were withdrawn at predetermined time intervals and every time replaced with $2 \mathrm{~mL}$ of fresh dissolution medium. The samples were analyzed by RP-HPLC-PDA method. The dissolution experiments were conducted in triplicate.

\section{Stability studies}

Stability studies were carried out on F7 containing 1.25\% w/w ZOL and hydroxypropyl methylcellulose (HPMC) E3. MDFs were packed in aluminum pouches, sealed, and stored at $40 \pm 2^{\circ} \mathrm{C}$ and $75 \pm 5 \% \mathrm{RH}$ for 6 months. The appearance, weight, and drug content properties of the MDFs were examined.

\section{Statistical analysis}

The results of experimental data were subjected to one-way ANOVA (using Fisher's LSD post hoc test) using SYSTAT software (SYSTAT Software Inc., San Jose, USA). The results with $\mathrm{p}<0.05$ were considered as of significant variance.

\section{RESULTS AND DISCUSSION}

In the present investigation, the MDFs were prepared using different film-forming agents, plasticizers, and different thickness and drug loads to study the effect of these variables on physicomechanical and drug release properties of MDFs. MDFs were prepared using Wet Film Applicator technique to ensure uniformity in film thickness throughout the whole area of films.

\section{Preparation of ZOL MDFs}

Initially, placebo MDFs were prepared with different polymers such as HPMC (E3, E5, and E15) methylcellulose, sodium carboxymethyl cellulose ( $\mathrm{Na} \mathrm{CMC}$ ), and sodium alginate using polyethylene glycol (PEG)-400 and glycerol as plasticizers and assessed for film forming capacities and appearance. Methylcellulose and sodium alginate showed poor film-forming capacities with MDFs not easily separable from glass plate after casting, whereas MDFs prepared using Na CMC and HPMC polymers were clear with good mechanical properties. Therefore, Na
CMC and HPMC were selected for further development. However, on drug loading, crystallization of ZOL was observed immediately with MDFs prepared using Na CMC. Besides, the MDFs were brittle and were not easily separable from the glass plate, whereas MDFs prepared with HPMC polymers were transparent and elegant in appearance with quick-drying rates and good mechanical properties. Hence, HPMC polymers with three viscosity grades (E3, E5, and E15) were selected for the development of ZOL MDFs. Different ZOL MDFs were prepared as per the formulae given in Table 1. A $5 \mathrm{~g}$ batch size of formulations gave approximately $90 \mathrm{~cm}^{2}$ film area.

\section{Morphological properties}

MDFs were visually tested for homogeneity, transparency, color, and smoothness. MDFs formulated with $2 \% \mathrm{w} / \mathrm{w}$ ZOL load were transparent initially but turned opaque within 10 days, which might be due to recrystallization of ZOL within the MDFs. To prevent the crystallization, PVP K30 as a solubilizing agent was added to formulations at $0.04 \% \mathrm{w} / \mathrm{w}$ level. However, crystallization of ZOL was still observed, and hence, further trails were made by decreasing the ZOL load to $1.25 \% \mathrm{w} / \mathrm{w}$. MDFs with $1.25 \% \mathrm{w} / \mathrm{w}$ ZOL load were clear and transparent with no crystallization even at the end of 6months. Hence, ZOL load of $1.25 \% \mathrm{w} / \mathrm{w}$ was chosen for further development. The photographs of ZOL MDFs with 2 and $1.25 \%$ w/w drug loads are shown in Fig. 1. The morphological characterization of MDFs was further established by visualizing MDFs under a binocular microscope (Olympus-CH20i) with magnification $\times 10$. The photomicrographs of MDFs are shown in Fig. 2 .

\section{DSC studies}

Crystallization of ZOL within the MDFs was further evaluated by DSC studies. DSC thermograms obtained for ZOL and selected ZOL MDFs (F6 and F7) are shown in Fig. 3. Thermogram of ZOL showed a solitary sharp melting endothermic peak at $141.6^{\circ} \mathrm{C}$, indicating that ZOL exists in a single crystalline state. Thermograms of ZOL MDFs demonstrate no or weak peak signals compared to pure $\mathrm{ZOL}$, and it might be due to

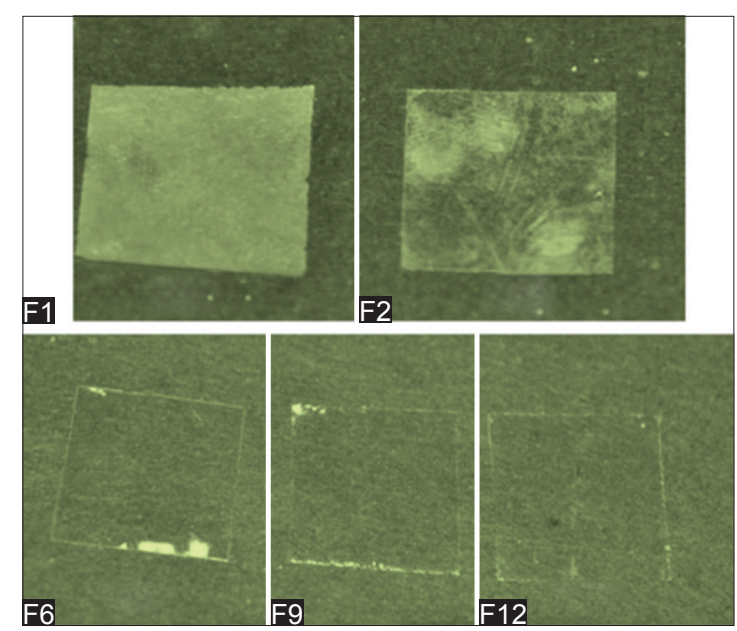

Fig. 1: Photographs of ZOL MDFS with 2\%w/w (F1 and F2) and $1.25 \% \mathrm{w} / \mathrm{w}$ (F6, F9, and F12) ZOL loads. (Coloring agent was not added to show the difference in appearance between crystallized and optimized films). ZOL: Zolmitriptan, MDF: Mouth dissolving films
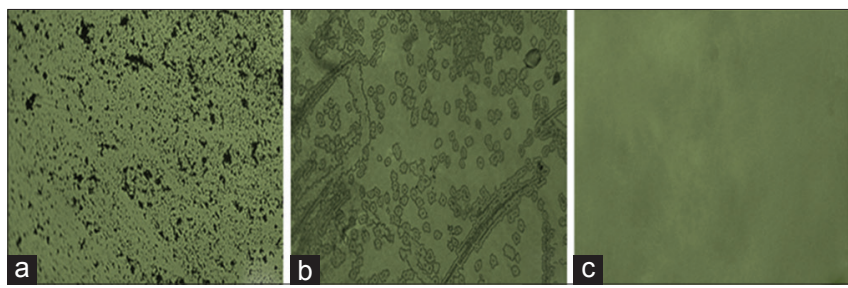

Fig. 2: Photomicrographs of (a) (zolmitriptan), (b) (F1), (c) (F6) 
molecular dispersion of ZOL within the MDFs. Furthermore, no extra peaks were observed in the thermograms indicating the absence of recrystallization of ZOL within the MDFs.

\section{X-RD studies}

ZOL and selected ZOL MDFs (F6 and F7) were subjected to X-RD studies to gain deeper insights into the crystallographic properties of ZOL in MDFs. ZOL demonstrated characteristic peaks at $19^{\circ}, 21.70^{\circ}, 23.65^{\circ}$, and $37.82^{\circ} 2 \theta$. The X-ray diffractograms of the ZOL MDFs (Fig. 4.) showed weak or no signals when compared to the characteristic peaks of ZOL. Overall, together with DSC data, the X-RD results clearly indicate that the ZOL was not in a crystalline state in MDFs.

\section{FT-IR studies}

The compatibility of ZOL with different excipients used in the formulation was evaluated using FT-IR studies, and FT-IR spectra of ZOL and MDFs (F7) are shown in Fig. 5. ZOL showed characteristic peaks at $3350 \mathrm{~cm}^{-1}$ (aromatic secondary amine N-H stretching), $2974 \mathrm{~cm}^{-1}$ (aromatic C-H stretching), $1736 \mathrm{~cm}^{-1}$ ( $\mathrm{C}=05$ member cyclic stretching), and $1259 \mathrm{~cm}^{-1}$ (C-N aliphatic amine stretching) wavenumbers. All these characteristic peaks of ZOL were retained in the MDFs and no shift in major peaks was observed, indicating that there is no interaction between ZOL and excipients in MDFs.

\section{Thickness}

The thickness of the MDFs was measured with screw gauge at different places of the whole film to evaluate the reproducibility of the preparation method. Around $90 \%$ of wet film thickness was lost during drying. Preparation of MDFs with wet film applicator brought

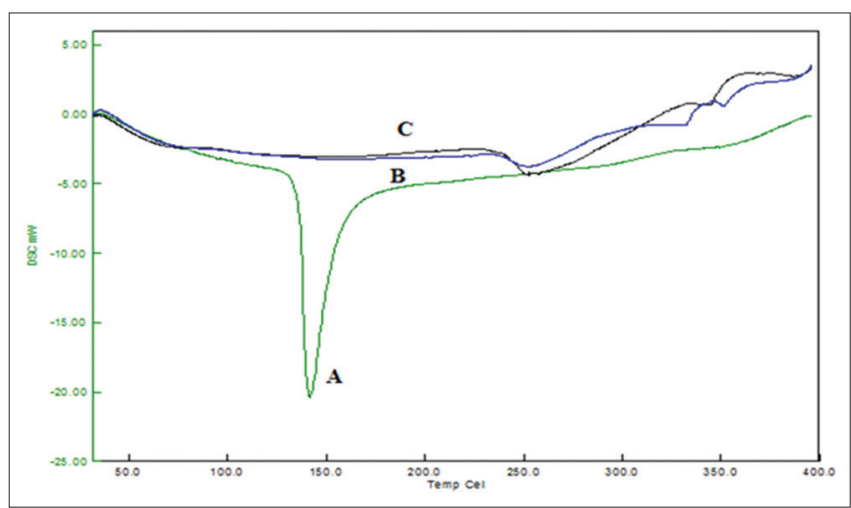

Fig. 3: Differential scanning calorimetry thermograms of Zolmitriptan (A), F6 (B), and F7 (C)

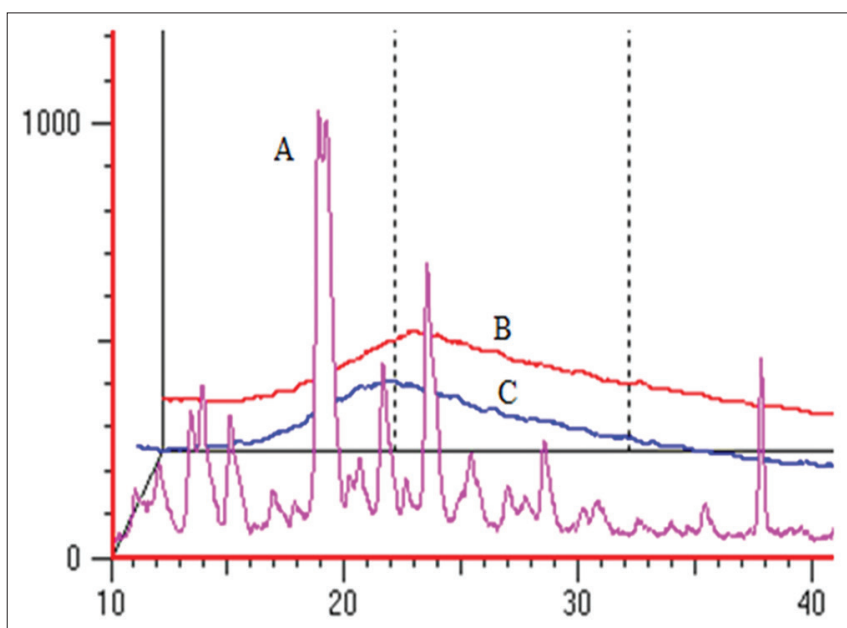

Fig. 4: X-ray diffractometry spectra of zolmitriptan (A), F6 (B) and F7 (C) in uniform thickness throughout the whole area of film ( $p>0.05)$. The results are given in Table 2. MDFs casted at 40 mil showed higher thickness $(100.00 \pm 0.00 \mu \mathrm{m}$ and $108.33 \pm 4.08 \mu \mathrm{m}$, respectively, for F1 A and F2 A) compared to MDFs casted at 30 mil $(70.00 \pm 0.00 \mu \mathrm{m}$ and $78.33 \pm 4.08 \mu \mathrm{m}$, respectively, for F1 and F 2). Among the polymers used, MDFs with HPMC E15 as film former showed higher thickness $(88.33 \pm 4.08 \mu \mathrm{m})$ compared to HMPC E3 $(68.33 \pm 4.08 \mu \mathrm{m})$ and E5 $(78.33 \pm 4.08 \mu \mathrm{m})$. Higher polymer viscosity as in the case with E15 resulted in lesser film area and subsequently MDFs with higher thickness.

\section{ZOL content}

Films of $1 \mathrm{~cm}^{2}$ were cut from different places $(\mathrm{n}=3)$ of the MDF, and ZOL content was estimated. The results are given in Table 2 . The results indicated a good uniformity of ZOL within the film ( $p>0.05$ ), and overall good solubilization of ZOL in MDFs was observed. MDFs casted at 40 mil thickness gave higher ZOL content values $\left(1.30 \pm 0.05 \mathrm{mg} / \mathrm{cm}^{2}\right.$ and $1.39 \pm 0.05 \mathrm{mg} / \mathrm{cm}^{2}$, respectively, for F1A and F2A) compared to MDFs casted at 30 mil thickness $\left(1.09 \pm 0.01 \mathrm{mg} / \mathrm{cm}^{2}\right.$ and $1.18 \pm 0.02 \mathrm{mg} / \mathrm{cm}^{2}$, respectively, for F1 and F2), which may be due to decrease in film area resulting in more drug entrapment within the small area of the film.

\section{Variation of mass}

Mass of $1 \mathrm{~cm}^{2}$ films cut from different batches was recorded on an electronic balance (Shimadzu-ATX224), and the results are given in Table 2. The same mass was obtained with three batches of films $(p>0.05)$ indicating reproducibility of preparation method and formulation.

\section{In vitro disintegration studies}

In the present investigation, disintegration time was assessed by drop and Petri dish methods, and the results are given in Table 2. From the results, it was found that thickness of films, polymer viscosities, and presence of solubilizing agents had affected the disintegration times of MDFs. A 2.82-fold decrease in disintegration times was observed

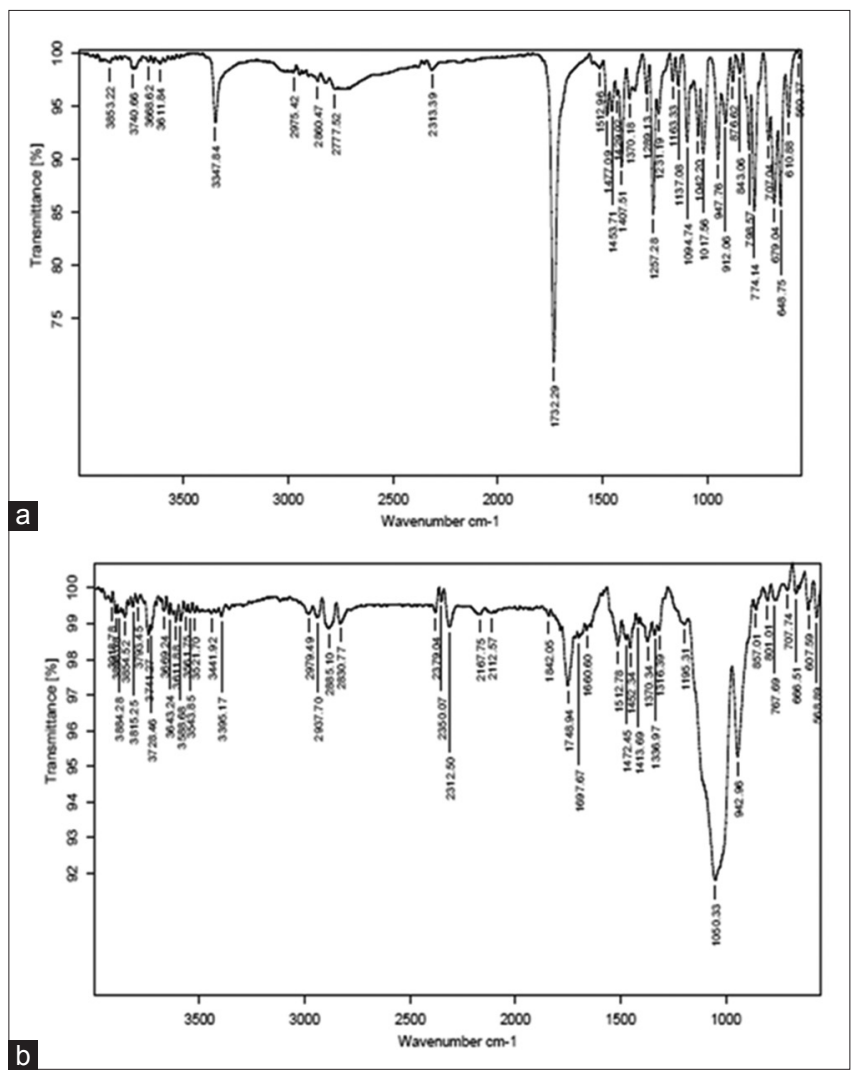

Fig. 5: Fourier-transform infrared spectra of zolmitriptan (a) and F7 (b) 
Table 2: Physicomechanical and chemical properties of ZOL MDFs

\begin{tabular}{|c|c|c|c|c|c|c|c|}
\hline \multirow[t]{2}{*}{ Formulations } & \multirow{2}{*}{$\begin{array}{l}\text { ZOL content* } \\
\left(\mathrm{mg} / \mathrm{cm}^{2}\right)\end{array}$} & \multirow[t]{2}{*}{ Thickness* $(\mu \mathrm{m})$} & \multirow[t]{2}{*}{ Mass variation* $(\mathrm{mg})$} & \multirow[t]{2}{*}{ \% Elongation } & \multirow{2}{*}{$\begin{array}{l}\text { Folding } \\
\text { endurance* }\end{array}$} & \multicolumn{2}{|c|}{ Disintegration time* (s) } \\
\hline & & & & & & Drop method & Petri dish method \\
\hline F1 & $1.09 \pm 0.01$ & $70.00 \pm 0.00$ & $4.06 \pm 0.05$ & $89.50 \pm 0.77$ & $106 \pm 0.58$ & $21.33 \pm 0.58$ & $30.00 \pm 0.00$ \\
\hline $\mathrm{F} 1(\mathrm{~A})$ & $1.30 \pm 0.05$ & $100.00 \pm 0.00$ & $5.48 \pm 0.46$ & $47.36 \pm 2.72$ & $34 \pm 1.53$ & $60.33 \pm 0.58$ & $82.33 \pm 0.58$ \\
\hline $\mathrm{F} 2$ & $1.18 \pm 0.02$ & $78.33 \pm 4.08$ & $4.35 \pm 0.30$ & $67.30 \pm 1.92$ & $74 \pm 1.53$ & $17.33 \pm 1.73$ & $27.67 \pm 0.58$ \\
\hline F2(A) & $1.39 \pm 0.02$ & $108.33 \pm 4.08$ & $5.67 \pm 0.58$ & $33.44 \pm 1.16$ & $16 \pm 1.15$ & $50.67 \pm 2.31$ & $75.67 \pm 0.58$ \\
\hline F3 & $1.06 \pm 0.08$ & $71.67 \pm 4.08$ & $4.11 \pm 0.08$ & $92.96 \pm 0.90$ & $109 \pm 1.00$ & $12.00 \pm 0.00$ & $24.00 \pm 1.00$ \\
\hline F4 & $1.08 \pm 0.05$ & $81.67 \pm 4.08$ & $4.78 \pm 0.33$ & $69.16 \pm 1.47$ & $78 \pm 1.53$ & $11.00 \pm 0.00$ & $22.33 \pm 0.58$ \\
\hline F5 & $0.75 \pm 0.00$ & $81.67 \pm 4.08$ & $4.67 \pm 0.21$ & $65.69 \pm 0.29$ & $72 \pm 1.15$ & $17.67 \pm 0.58$ & $28.67 \pm 1.15$ \\
\hline F6 & $0.70 \pm 0.01$ & $68.33 \pm 4.08$ & $3.52 \pm 0.36$ & $74.60 \pm 0.69$ & $108 \pm 1.53$ & $23.00 \pm 0.00$ & $32.33 \pm 0.58$ \\
\hline F7 & $0.75 \pm 0.02$ & $70.00 \pm 0.00$ & $4.00 \pm 0.00$ & $99.68 \pm 0.77$ & $115 \pm 1.73$ & $11.00 \pm 0.00$ & $24.67 \pm 0.58$ \\
\hline F8 & $0.76 \pm 0.02$ & $70.00 \pm 0.00$ & $4.00 \pm 0.00$ & $97.36 \pm 0.32$ & $114 \pm 0.58$ & $12.66 \pm 0.58$ & $26.00 \pm 0.00$ \\
\hline F9 & $0.71 \pm 0.00$ & $78.33 \pm 4.08$ & $4.32 \pm 0.17$ & $80.14 \pm 0.24$ & $92 \pm 0.58$ & $28.00 \pm 0.00$ & $38.00 \pm 0.00$ \\
\hline F10 & $0.76 \pm 0.03$ & $80.00 \pm 0.00$ & $4.35 \pm 0.29$ & $76.18 \pm 0.31$ & $86 \pm 1.15$ & $18.67 \pm 0.58$ & $34.00 \pm 0.00$ \\
\hline F11 & $0.75 \pm 0.02$ & $80.00 \pm 0.00$ & $4.41 \pm 0.09$ & $72.70 \pm 0.69$ & $82 \pm 2.25$ & $20.00 \pm 0.00$ & $35.00 \pm 0.00$ \\
\hline F12 & $0.79 \pm 0.01$ & $88.33 \pm 4.08$ & $4.95 \pm 0.06$ & $95.04 \pm 0.07$ & $88 \pm 1.00$ & $33.33 \pm 0.58$ & $42.67 \pm 0.58$ \\
\hline
\end{tabular}

* Values represented are mean \pm SD ( $n=3$ ). ZOL: Zolmitriptan, MDF: Mouth dissolving films, SD: Standard deviation

with MDFs casted at 30 mil thickness compared to 40 mil films. Faster disintegration of 30 mil films can be attributed to lesser film thickness compared to $40 \mathrm{mil}$ films. The results also indicate that an increase in polymer viscosity delayed the disintegration of MDFs. HPMC E15 formulations showed significantly slower disintegration times $(\mathrm{p}<0.05)$ compared to E5 and E3 formulations. Addition of PVP K30 and SLS as solubilizing agents to formulations resulted in faster disintegration of MDFs, and about 2.09- and 1.81-fold decrease in disintegration times was observed for PVP and SLS formulations, respectively, compared to MDFs without them. The images of MDF (F7) disintegration by drop and Petri dish methods are shown in Fig. 6a and b, respectively.

\section{Tensile strength and percentage elongation}

In the present investigation, the effect of polymer viscosities, film thickness, plasticizers, and solubilizing agents on mechanical properties of ZOL MDFs was studied. The tensile strength of MDFs was measured using Mini Tech Tensiometer-UTM9051 and the tensile strength profiles are shown in Fig. 7. From the results obtained, it was found that MDFs casted at 40 mil showed low tensile strength compared to MDFs of 30 mil thickness showing the inverse relation between tensile strength and film thickness (Fig. 7a). Formulations prepared with HPMC E15 showed higher tensile strength compared to E5 and E3, indicating that an increase in polymer viscosity resulted in an increase in tensile strength of the films (Fig. 7c). MDFs with glycerol as plasticizer showed low tensile strength values compared to MDFs with PEG-400 (Fig. 7b). No significant difference in tensile strength of MDFs was observed with the addition of solubilizing agents to the formulations.

Percentage elongation of films was computed form TestBench-II software during tensile strength measurements and the results are given in Table 2. MDFs casted at 40 mil thickness (F1A) showed less percentage elongation (47.36 \pm 2.72 ) compared 30 mil films (F1, $89.50 \pm 0.77$ ). Among the different polymers used, higher percentage elongation was obtained with HPMC E15 films (F12, $95.04 \pm 0.07$ ) compared to E3 $(\mathrm{F} 6,74.60 \pm 0.69)$ and E5 (F9, $80.14 \pm 0.24)$ films. Films prepared with PEG-400 as a plasticizer (F1) showed higher percentage elongation values $(89.50 \pm 0.77)$ compared to films prepared with glycerol ( $F 2,67.30 \pm 1.92$ ). Overall, from the results, it can be inferred that polymer viscosities, plasticizers, and film thickness have a significant impact on the mechanical properties of MDFs [15]. MDFs with glycerol as plasticizer and MDFs casted at 40 mil thickness were brittle with poor mechanical properties compared to other formulations. Overall, MDFs prepared with HPMC E3 containing PEG 400 as plasticizer casted at 30mil thickness showed moderate tensile strength and high percentage elongation.

\section{Folding endurance}

Along with tensile strength, folding endurance demonstrates the resistance of film toward mechanical forces during packing or transport. MDFs prepared with glycerol and MDFs casted at 40 mil thickness
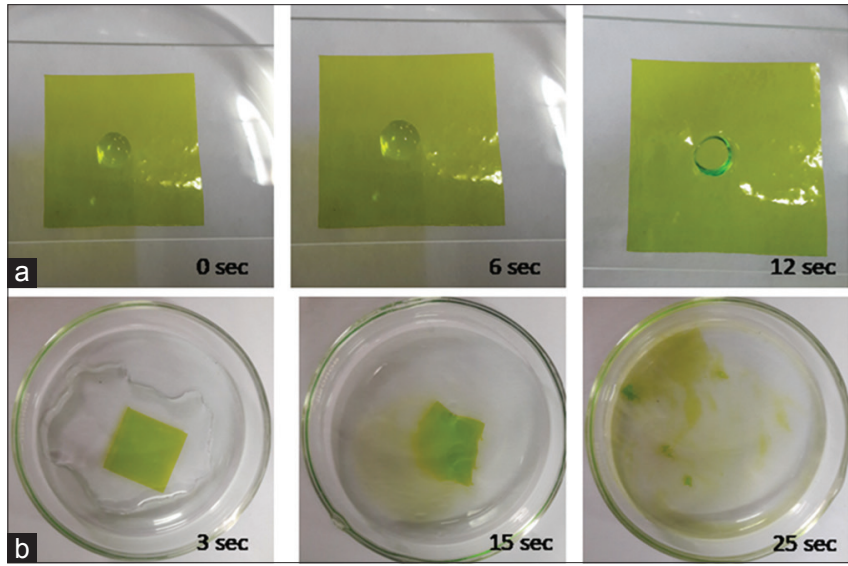

Fig. 6: In vitro disintegration of ZOL MDFS (F7) by drop method (a) and Petri dish method (b). ZOL: Zolmitriptan, MDF: Mouth dissolving films

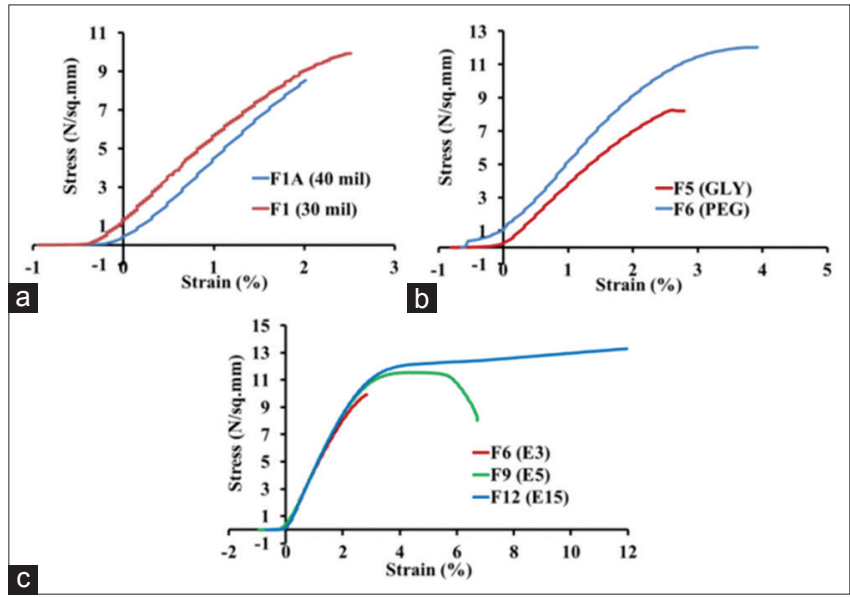

Fig. 7: Effect of film thicknesses (a), plasticizers (b) and polymer viscosities (c) on tensile strength of films

showed low folding endurance values compared to other formulations. The results are shown in Table 2.

\section{In vitro drug release studies}

In the present investigation, ZOL release from MDFs was evaluated using USP Type-V dissolution rate testing apparatus. $500 \mathrm{~mL}$ of artificial saliva was used as dissolution medium to mimic the in vivo conditions. The 
effect of various formulation variables such as thickness, plasticizers, polymer viscosities, and solubilizing agents on ZOL release from MDFs was studied.

Initially, to study the influence of plasticizers and film thickness on ZOL release rates from MDFs, formulations were prepared using HPMC E3 as film-forming agent, PEG-400, and glycerol as plasticizers and casted at 30 and 40 mil thickness. The cumulative percentage ZOL released at the end of $5 \mathrm{~s}$ is $5.62 \pm 0.95,3.50 \pm 0.42,3.91 \pm 0.15$, and $3.60 \pm 0.21$ for F1, F1 (A), F2, and F2 (A), respectively. Complete ZOL release was obtained at $300 \mathrm{~s}$ and $240 \mathrm{~s}$ for F1 and F2, respectively, whereas F1 (A) showed only $67.13 \pm 0.56 \%$ release at the end of 300 s and F2 (A) showed only $56.9 \pm 1.93 \%$ ZOL release at the end of $240 \mathrm{~s}$ (Fig. 8a).

The ZOL release from MDFs casted at 30 mil thickness was significantly higher $(\mathrm{p}<0.05)$ when compared to MDFs of 40 mil thickness. The higher film thickness resulted in more time being taken by the films to disintegrate and hence resulted in slower ZOL release from MDFs [16]. Hence, a film thickness of 30 mil was selected for further formulation studies. Among the plasticizers used, formulations with glycerol showed slightly faster ZOL release compared to the formulation containing PEG 400. However, PEG 400 was selected as a plasticizer for further development based on its superior mechanical properties compared to glycerol.

Studies were continued to evaluate the effect of polymers viscosities on ZOL release from MDFs. Formulations were prepared with 30 mil thickness using PEG-400 as a plasticizer and HPMC E3, E5, and E15 as film formers. The cumulative percentage ZOL released at the end of $5 \mathrm{~s}$ is $17.64 \pm 1.20,15.62 \pm 0.71$ and $10.61 \pm 0.36$ for F6, F9, and F12, respectively. Complete ZOL release was obtained at 300, 420, and $600 \mathrm{~s}$, respectively (Fig. 8b). The ZOL release from F6 (only E3) is significantly higher $(\mathrm{p}<0.05)$ when compared to F9 (only E5) and F12 (only E15). Faster ZOL release rates observed with HPMC E3 polymer can be attributed to the low viscosity of E3 compared to E5 and E15.
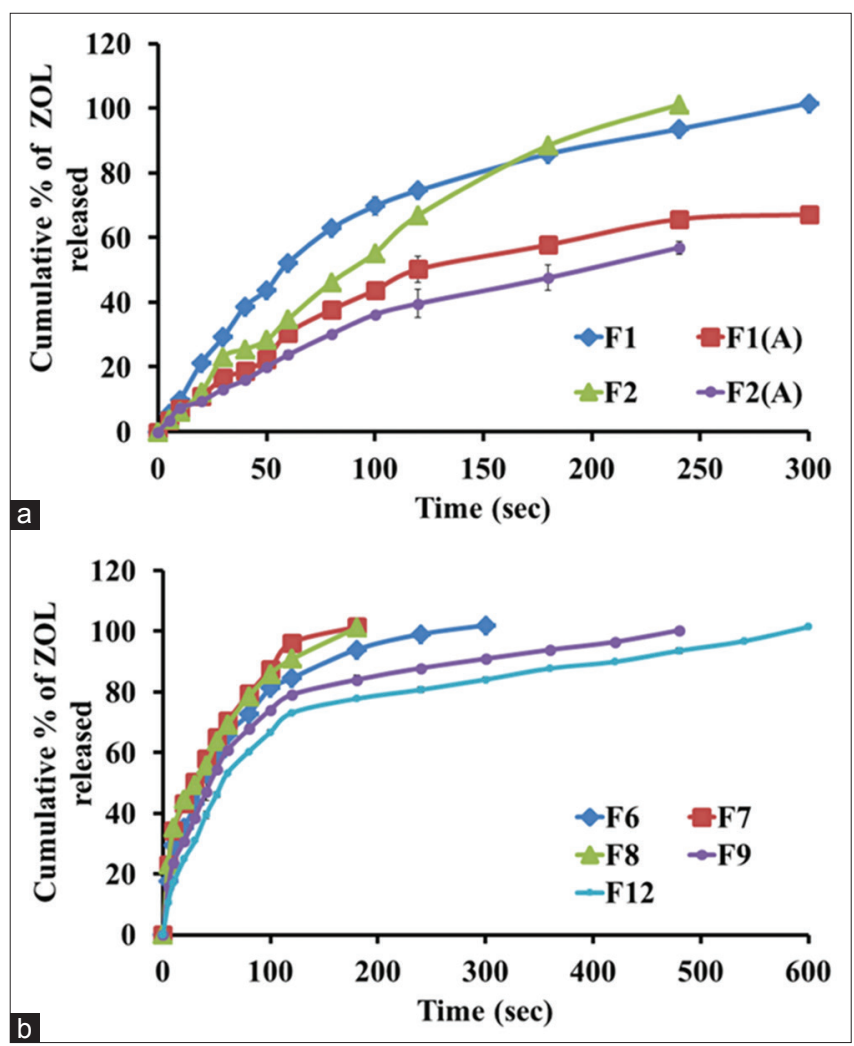

Fig. 8: Effect of film thickness, plasticizers (a), polymer viscosities, and solubilizing agents (b) on in vitro drug release from the films $(n=3)$
Overall, the order of percentage ZOL release from MDFs was found to be $\mathrm{F} 6>\mathrm{F} 9>\mathrm{F} 12$.

Further, the effect of solubilizing agents on ZOL release rates was also studied. Both PVP K30 and SLS were added to formulations at 0.04\% level. The cumulative percent ZOL release at the end of $5 \mathrm{~s}$ for MDFs with PVP K30 (F7) and SLS (F8) was $23.03 \pm 0.86$ and $23.16 \pm 0.59$, respectively (Fig. 8b). Complete ZOL release was obtained at $180 \mathrm{~s}$ for both the formulations. Addition of solubilizing agents resulted in faster ZOL release rates and about a 1.66- and 1.53-fold increase in ZOL release rates was obtained for F7 and F8, respectively, compared to MDFs without them.

Overall, from the in vitro drug release studies, it can be concluded that film thickness, polymer viscosities, and solubilising agents had a significant impact on ZOL release from MDFs [16]. Among the formulations prepared, F7 formulation showed superior ZOL release rates along with good physicomechanical properties compared to remaining formulation

\section{Drug release kinetics}

To better understand the release profiles obtained with ZOL MDFs formulations, the drug release data obtained at different time points were fitted into kinetic models such as first order [17] and Higuchi model [18].

The first-order release rate constant " $\mathrm{k}$ " $\left(\mathrm{s}^{-1}\right)$ values were higher for F1 $\left(0.011 \mathrm{~s}^{-1}\right)$ and F2 $\left(0.011 \mathrm{~s}^{-1}\right)$ compared to F1 (A) $\left(0.005 \mathrm{~s}^{-1}\right)$ and F2 (A) $\left(0.004 \mathrm{~s}^{-1}\right)$, respectively. A 2.2 - and 2.75 -fold increase in " $\mathrm{k}$ " values was observed for F1 and F2 with that of F1 (A) and F2 (A). The " $k$ " values were higher for HPMC E3 (F6) formulations compared to E5 (F9) and E15 (F12) formulations. A 1.36- and 1.66-fold increase in " $\mathrm{k}$ " values was obtained for F6 $\left(0.015 \mathrm{~s}^{-1}\right)$ compared to F9 $\left(0.011 \mathrm{~s}^{-1}\right)$ and F12 $\left(0.009 \mathrm{~s}^{-1}\right)$. When compared to F6 (only E3), the first-order release rate constant " $\mathrm{k}$ " values were significantly higher for F7 and F8 containing PVP K 30 and SLS. A 1.66- and 1.53-fold increase in " $k$ " values for F7 $\left(0.025 \mathrm{~s}^{-1}\right)$ and F8 $\left(0.023 \mathrm{~s}^{-1}\right)$ was observed when compared to F6. The Higuchi square root model of all MDFs showed higher correlation coefficient values (0.899-0.993) indicating diffusion as a release mechanism.

\section{Stability studies}

Stability studies were carried out for F7 formulation containing $1.25 \% \mathrm{w} / \mathrm{w}$ ZOL with HPMC E3 and PVP K30. MDFs were stored at $40 \pm$ $2^{\circ} \mathrm{C}$ with a relative humidity of $75 \pm 5 \%$ for 6 months. The appearance, weight variation, and drug content of the MDFs were examined. The appearance of MDFs remained unchanged throughout the studies, and no crystallization was observed. There is no statistically significant change ( $p>0.05$ ) observed in weight of MDFs. F7 showed 95-99\% of ZOL content after 6 months, indicating that the ZOL was stable in MDFs.

\section{CONCLUSION}

From this investigation, it can be concluded that ZOL can be successfully formulated as MDFs. From the results, it was observed that film thickness and plasticizers had a significant impact on the mechanical properties of MDFs. In addition, polymer viscosity was found to play a crucial role in ZOL release rates along with the physicomechanical properties. Faster drug release rates obtained from MDFs may provide a quick onset of action with enhanced oral bioavailability and therapeutic efficacy compared to current marketed formulations such as IR and ODTs.

\section{ACKNOWLEDGMENT}

The authors are thankful to AICTE, New Delhi, for funding the research work, Mylan Laboratories, Hyderabad, for providing ZOL, and Colorcon, India, for providing HPMC samples and Siddhartha Academy of General and Technical Education, Vijayawada, for providing necessary facilities to carry out this research work. 


\section{AUTHORS' CONTRIBUTIONS}

Buchi N. Nalluri: Supervised and provided guidance for this research project and helped with the preparation of the manuscript. Sudhir Maddela: Conceptualized the article, compiled literature and data. and drafted the manuscript.

\section{CONFLICTS OF INTEREST}

The authors declare that they have no conflicts of interests.

\section{REFERENCES}

1. Lopez FL, Ernest TB, Tule C, Gull MO. Formulation approaches to paediatric oral drug delivery: Benefits and limitations of current platforms. Expert Opin Drug Deliv 2015;12:1727-40.

2. Ivanovska V, Rademaker CM, Van Dig KL, Teeuwisse AK. Paediatric drug formulations: Areview of challenges and progress. Paediatrics 2014; 134:361-72.

3. Parul S, Anoop K, Pankaj S, Sharad V. Fast disintegrating oral films: A recent trend of drug delivery. Int J Drug Dev Res 2012;4:80-94.

4. Sastry SV, Nyshadham JR, Fix JA. Recent technological advances in oral drug delivery: A review. Pharm Sci Tech Today 2000;3:138-45.

5. Arun A, Chandra A, Sharma V, Pathak K. Fast dissolving oral films: An innovative drug delivery system and dosage form. Int J Chem Tech Res 2010;2:576-83.

6. Dixit AS, Kulakarni PK, Reddy SC. Methotrexate fast disintegrating tablet as a dosage form for dysphagia patients. Int J Pharm Pharm Sci 2014;6:217-5.

7. Desu P, Sahu M. Formulation and evaluation of fast dissolving films of zolmitriptan. Int Res J Pharm 2012;3:373-76.
8. Natarajan R, Sravanthi CH, Saranya V, Rajendran NN. Effect of super disintegrants on oral disintegrating tablets of zolmitriptan. Int J Pharm Ind Res 2014;4:57-62.

9. Hirokazu O, Hirohisa T, Kotaro I, Kazumi D. Development of polymer film dosage form of lidocaine for buccal administration. J Controll Release 2001;77:253-60.

10. Nair AB, Kumria R, Harsha S, Mahesh A, Bandar E, Alhaider IA. In vitro techniques to evaluate buccal films. J Controll Release 2013;116:10-21

11. Garsuch V, Breitkreutz J. Comparitive investigations on different polymers for the preparation of fast-dissolving oral films. J Pharm Pharm 2010;62:539-45.

12. El-Setouhy DA, El-Malak NS. Formulation of a novel tianeptine sodium orodispersible film. AAPS Pharm Sci Tech 2010;11:1018-25.

13. Gavaskar B, Kumar SV, Sharan G, Madhusudan Y. Overview on fast dissolving films. Int J Pharm Pharm Sci 2010;2:29-33.

14. Okamoto H, Taguchi H, Iida K, Danjo K. Development of polymer film dosage forms of lidocaine for buccal administration. I. Penetration rate and release rate. J Control Release 2001;77:253-60.

15. Lai KL, Yuan F, Hao H, Li Q, Shuai Z, Li HY, et al. Orally dissolving film for sublingual and buccal delivery of ropinrole. Colloids Surf B 2018; 163:9-18.

16. Takeuchi Y, Umemura K, Tahara K, Takeuchi H. Formulation design of hydroxypropyl cellulose films for use as orally disintegrating dosage forms. J Drug Deliv Sci Tech 2018;46:93-100.

17. Lapidus H, Lordi NG. Drug release from compressed hydrophilic matrices. J Pharm Sci 1996;55:840-3.

18. Higuchi T. Mechanism of sustained action medication. Theoretical analysis of rate of release solid drugs dispersed in solid matrices. J Pharm Sci 1963;52:1145-8. 